\title{
Chiapas, sede del V Congreso Nacional de Hospitales Regionales de Alta Especialidad del país
}

\author{
Fernando Tapia-Garduño* \\ Dirección de Planeación, Enseñanza e Investigación del Centro Regional de Alta Especialidad, Chiapas. México
}

- Participaron los hospitales regionales de alta especialidad de Oaxaca, Ciudad Victoria, la Península de Yucatán, el Bajío e Ixtapaluca, así como el Centro Regional de Alta Especialidad y sus dos unidades aplicativas: Ciudad Salud en Tapachula y el Hospital de Especialidades Pediátricas de Tuxtla Gutiérrez, como anfitriones en este año.

- Además, se celebró el Día Nacional de la Donación y Trasplante de Órganos; Chiapas será la primera entidad en conmemorar este día.

San Cristóbal de Las Casas, Chiapas. Al iniciar el 5. ${ }^{\circ}$ Congreso Nacional de los Hospitales Regionales de Alta Especialidad y $4 .{ }^{a}$ Reunión Nacional de Auditoria Clínica y Mejoría de la Calidad de la Atención Médica, el director general de la Coordinación de los Hospitales Regionales de Alta Especialidad de país (septiembre 25 a 27 del 2019), Manuel de la Llata Romero, consideró que los hospitales regionales de alta especialidad (HRAE) actualmente son espacios de atención médica especializada que evitan gastos onerosos a familias de la región y de igual manera traslados al centro y norte del país.

«Chiapas es el único Estado con dos HRAE. Están formados por una masa crítica de especialistas que ofrecen servicios médicos que cambian vidas. Estos dos hospitales lo han logrado con creces», aseguró.
Añadió de la Llata Romero que en estos años Ios:HRAE tienen un papel preponderante; el HRAE del Bajío es un ejemplo en el país en materia de trasplantês. Hay aportes en investigación como las realizadas en E. E्iudad Salud de Tapachula, además de programas y pröyectos que nos llenan de orgullo por su contribución alt desarrollo del país.

El secretario de Salud de Chiapas, José Manǚ Cruz Castellanos, en representación del gobernadorónutilio Escandón Cadenas, reconoció la aportación de tejpersonal de salud de los hospitales de especialidades como una plataforma integral que cambia vidas.

«En Chiapas estamos comprometidos con eficientar y transparentar la salud pública en la población, tal y como ha sido la instrucción del gobernador del $\bar{E}$ Estado. Y desde aquí se gestan aportes que, sin dudąi serán agentes de cambio en el país».

Al dar la bienvenida, la directora del Centro Rêgional de Alta Especialidad de Chiapas, Concepción guez González, explicó que «durante tres días èt Congreso brindará la oportunidad de asistir a conferencias magistrales y a exposiciones de destacados investigadores, médicos especialistas y funcionarios. Se un evento que promueve la actualización, la prófesionalización y la especialización en temáticas cơmo la

\section{Correspondence:}

*Fernando Tapia-Garduño

E-mail: ferfreud@ hotmail.com
Date of reception: 30-09-2019

Date of acceptance: 04-10-2019

DOI: 10.24875/HMCM.M19000010 
atención médica, los desafíos de la investigación y las auditorías clínicas».

«Es un foro que facilita la interacción, el intercambio y la colaboración entre investigadores, profesores, trabajadores de la salud y servidores públicos, y que además nos da la oportunidad de estrechar vínculos con expertos de otros Estados del país», subrayó la también especialista.

En el primer día de actividades se abordaron las temáticas de: atención médica pediátrica oncológica, atención médica enfocada a trasplantes por el HRAE de Oaxaca y desafíos de la investigación, coordinado por la Comisión Coordinadora de Institutos Nacionales de Salud y Hospitales de Alta Especialidad, en el que participaron los HRAE de Ixtapaluca, Ciudad Victoria y Ciudad Salud, en tanto que el Hospital de Especialidades Pediátricas de Tuxtla Gutiérrez, presentó el proyecto de hematooncología.

En este marco, Cruz Castellanos disertó la ponencia magistral denominada «Transformación del Sistema de Salud en Chiapas», ofreciendo pormenores de los avances, y un modelo de salud con los retos y la ruta de construcción y mejoramientos continuo de los servicios de salud.

Asistieron como invitados de honor: Simón Kawa Kasarik, director general de la Coordinación de los Institutos Nacionales de Salud; Rodolfo Cano Jiménez, director de Investigación en Salud; la presidenta municipal de San Cristóbal de Las Casas, Jerónima Toledo Villalobos, y Alberto Cundapí Núñez, director general del Instituto de Seguridad Social de los Trabajadores del Estado.

El marco de este congreso durante el segundo día de actividades sirvió de sede para conmemorar el Día Nacional de la Donación de Órganos y Tejidos.

El gobernador Rutilio Escandón Cadenas encabezó la celebración del Día Nacional de la Donación y Trasplante de Órganos, en el municipio de San Cristóbal de Las Casas, donde refrendó su compromiso de promover esta acción altruista entre la sociedad chiapaneca, a fin de sensibilizar a más personas y ofrecer mayores oportunidades a quienes están a la espera de un trasplante.

«Donar no solamente es dar a un desconocido una parte de tus órganos o tejidos, es seguir viviendo a

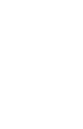

través de otra persona y que, incluso en vida, ’es una acción que enaltece y fortalece. Tenemos que ha્çcer un verdadero ejército de comunicación, información y conocimientos para que toda la ciudadanía sepa que donar solo trae como consecuencia acciones y conductas positivas para beneficiar a quienes lo necesitan», expresó.

En el evento, que por primera vez se realiza en Chiapas, el mandatario dio a conocer que cada año máš de 20 mil personas están esperando una donación y que lamentablemente no se alcanza a abastecer ni siquiera la mitad, por ello refrendó el compromiso de suğgobierno de trabajar para que más habitantes de Cthiapas, quienes se distinguen por su fraternidad, se unaña esta noble causa.

«Llegará el momento en que tengamos reservẫ de órganos para auxiliar a la gente, por ello buscaremos una forma de inculcar en más habitantes la cultură de la donación, no solamente expresarlo en la liceñcia de manejo sino en otros documentos que tenga \$oda la ciudadanía», agregó.

El director general del Centro Nacional de Traspîantes, José Salvador Aburto Morales, celebró que eñ estas tareas se cuente no solo con la participacióß de la sociedad, sino con la voluntad política de las inștituciones gubernamentales y civiles, ya que la acción de donar órganos y tejidos es una decisión voluntarria que debe de estar notificada en vida.

«En México se está trabajando intensamente para tener un sistema nacional de donación y trasplantes dê forma equitativa y transparente, al que todos, sin disîtnción, tengan acceso. Por ello, invito a toda la sociejdad a intensificar las acciones de información e impülsar la cultura de donación de órganos y tejidos, porque cuando sembramos ayuda, cosechamos vida», manifiestó.

Por su parte, la titular de la Unidad de Vinculăción y Participación Social de la Secretaría de Salud Federal, Mónica Mieres Hermosillo, en representación dețsecretario Dr. Jorge Alcocer Varela, destacó la necesiegad de fortalecer la contribución de la sociedad para lograr un Sistema de Salud Integral al tiempo de precisartel interés de la Federación de impulsar una normạtividad transparente y eficiente para mejorar el procéso de trasplante y su tratamiento, dentro de un marco dientífico, ético y legal. 
Al tomar la palabra, el titular de la Comisión Coordinadora de Institutos Nacionales de Salud y Hospitales de Alta Especialidad, Alejandro Mohar Betancourt, destacó el potencial de Chiapas para ser un verdadero líder en materia de trasplante de órganos, aunado a su compromiso de trabajar de la mano con la Federación para el fortalecimiento de las unidades de trasplante y del HRAE.

Al dar la bienvenida a especialistas en materia de donación de órganos, el Secretario de Salud del Estado, José Manuel Cruz Castellanos, comentó que con más avances en la ciencia y la investigación se logran buenos resultados en la atención a la salud, por ello se ha fortalecido el Centro Estatal de Trasplantes, con'el propósito de fomentar la donación multiorgánica.

En su turno el presidente de la Asociación Civil Ämigos Prorenal, Dona Vida, Recibe Esperanza, Gonzalo Rodríguez Pinal, compartió su experiencia al ser donador dé riñón hace cinco años y exhortó a las y los chiapanecos convertirse en grandes donadores en vida para salvaryjidas.

Finalmente se hizo entrega de reconocimiento ${ }^{\sigma} p o r$ la organización de este $V$ Congreso Nacional de los Hospitales Regionales de Alta Especialidad a la Dra. Concepción Domínguez González, Directora Genègal del Centro Regional de Alta Especialidad de Chiapas. 